\title{
Tomasz Człapiński
}

On the existence of generalized solutions of nonlinear first order partial differential-functional equations in two independent variables

Czechoslovak Mathematical Journal, Vol. 41 (1991), No. 3, 490-506

Persistent URL: http://dml.cz/dmlcz/102483

\section{Terms of use:}

(C) Institute of Mathematics AS CR, 1991

Institute of Mathematics of the Czech Academy of Sciences provides access to digitized documents strictly for personal use. Each copy of any part of this document must contain these Terms of use.

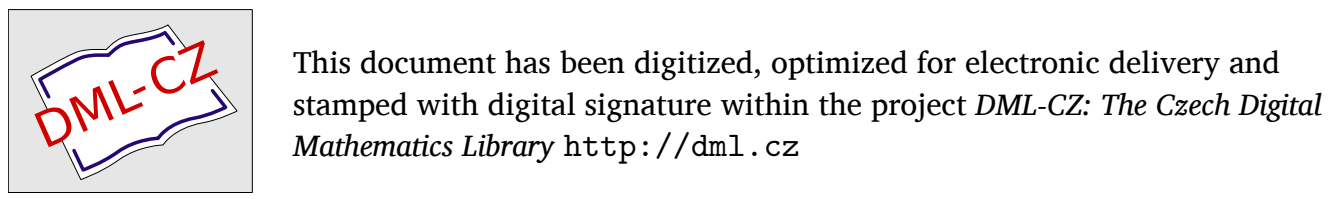




\section{ON THE EXISTENCE OF GENERALIZED SOLUTIONS \\ OF NONLINEAR FIRST ORDER PARTIAL \\ DIFFERENTIAL-FUNCTIONAL EQUATIONS \\ IN TWO INDEPENDENT VARIABLES}

Tomasz Czlapiński, Gdańsk

(Received June 11, 1990)

\section{INTRODUCTION}

Let $a_{0}>0, B=\left[-b_{0}, 0\right] \times\left[-b_{1}, b_{1}\right]$, where $b_{0}, b_{1} \in R_{+}, R_{+}=[0,+\infty)$. For any function $z:\left[-b_{0}, a_{0}\right] \times R \rightarrow R$ and for a fixed $(x, y) \in\left[0, a_{0}\right] \times R$ we define the function $z_{(x, y)}: B \rightarrow R$ by $z_{(x, y)}(t, s)=z(x+t, y+s),(t, s) \in B$. For any metric spaces $X, Y$ by $C(X, Y)$ we denote the set of all continuous functions defined on $X$ and taking values in $Y$.

Suppose that $f:\left[0, a_{0}\right] \times R \times R \times C(B, R) \times R \rightarrow R, \varphi:\left[-b_{0}, 0\right] \times R \rightarrow R$ and let us consider the following Cauchy problem for the nonlinear differentialfunctional equation of the first order

$$
\begin{aligned}
& D_{x} z(x, y)=f\left(x, y, z(x, y), z_{(x, y)}, D_{y} z(x, y)\right), \\
& z(x, y)=\varphi(x, y), \quad(x, y) \in\left[-b_{0}, 0\right] \times R .
\end{aligned}
$$

A function $u \in C\left(\left[-b_{0}, a_{0}\right] \times R, R\right)$ is a generalized solution of (1), (2) if

(i) $u$ satisfies the Lipschitz condition on $\left[0, a_{0}\right] \times R$,

(ii) there exists a function $\lambda \in C\left(\left(0, a_{0}\right], R_{+}\right)$such that $l^{-2}[u(x, y+l)-$ $-2 u(x, y)+u(x, y-l)] \leqq \lambda(x)$, for $(x, y) \in\left(0, a_{0}\right] \times R, l \in R, l \neq 0$,

(iii) $u$ satisfies (1) a.e. ("almost everywhere") on $\left[0, a_{0}\right] \times R$ and the initial condition (2) for all $(x, y) \in\left[-b_{0}, 0\right] \times R$.

Remark 1. If we omit the condition (ii) in the above definition, then the solution is not unique. An adequate example for $f$ without a functional argument is given in [16].

Generalized solutions of nonlinear first order partial differential equations have been investigated in a large number of papers by various authors. Theorems of existence, uniqueness and continuous dependence upon Cauchy or boundary data for quasilinear systems have been given by L. Cesari [7], [8], P. Bassanini [1] - [3] and P. Pucci [18]. Quasilinear differential-integral systems and systems with a retarded argument are considered in [4], [13], [14]. Nonlinear differential equations 
have been studied by M. Cinquini-Cibrario, S. Cinquini [9]. Generalized solutions of quasilinear and nonlinear equations with operators of the Volterra type are investigated in [20]-[22]. Additional bibliographical information may be found in [19].

Generalized solutions of nonlinear equations are also investigated in the case when assumptions for given functions are sufficient for existence of classical solutions (of class $C^{1}$ ). For classical solutions we can prove only a local existence and therefore to obtain theorems of a global existence we need generalized solutions. Theorems of this type have been given by S. N. Kruzhkov [16] and for equations with a retarded argument by Z. Kamont, S. Zacharek [15].

Classical solutions of nonlinear differential-functional equations or equations with a retarded argument are discussed in [5], [6], [10]- [12].

In this paper we prove the global existence of generalized solutions of (1), (2) extending the results of paper [16]. The proof is based on the difference method (see also $[17]$ ).

\section{ASSUMPTIONS AND DEFINITIONS}

We denote by $C_{0+L}(B, R)$ a set of all continuous functions from $B$ to $R$ which satisfy the Lipschitz condition on $B$. Furthermore, for any $t \in R_{+}$let $C_{0+L}(B, R, t)=$ $=\left\{u \in C_{0+L}(B, R):\|u\|_{0+L}=\|u\|_{0}+\|u\|_{L} \leqq t\right\}$, where

$$
\begin{aligned}
& \|u\|_{0}=\sup \{|u(s, v)|:(s, v) \in B\}, \\
& \|u\|_{L}=\sup \left\{[|s-\bar{s}|+|v-\bar{v}|]^{-1}|u(s, v)-u(\bar{s}, \bar{v})|:(s, v),(\bar{s}, \bar{v}) \in B\right\} .
\end{aligned}
$$

Suppose that the function $f:\left[0, a_{0}\right] \times R \times R \times C(B, R) \times R \rightarrow R$ of the variables $(x, y, p, w, q)$ is of class $C^{2}$. By $D_{y} f, D_{p} f, D_{q} f, D_{y y}^{2} f, D_{y q}^{2} f, D_{y p}^{2} f, D_{p p}^{2} f$, $D_{q p}^{2} f, D_{q q}^{2} f$ we denote first or second order partial derivatives of $f . D_{w} f$ is the Frechet derivative of $f$ i.e. $D_{w} f(x, y, p, w, q) \in \mathscr{L}(C(B, R), R)$, where $\mathscr{L}(X, Y)$ denotes a set of all linear operators from $X$ to $Y$. Symbols $D_{y w}^{2} f, D_{p w}^{2} f, D_{q w}^{2} f$ have the same meaning as $D_{w} f$ while $D_{w w}^{2} f$ denotes the second order Frechet derivative i.e. $D_{w w}^{2} f(x, y, p, w, q) \in \mathscr{L}(C(B, R), \mathscr{L}(C(B, R), R))$.

\section{Assumption H. Suppose that}

$1^{\circ} \varphi \in C\left(\left[-b_{0}, 0\right] \times R, R\right)$ and there are constants $\tilde{M}, \tilde{L} \in R_{+}$such that for all $(x, y),(x, \bar{y}) \in\left[-b_{0}, 0\right] \times R$ we have

$$
|\varphi(x, y)| \leqq \tilde{M}, \quad|\varphi(x, y)-\varphi(x, \bar{y})| \leqq \tilde{L}|y-\bar{y}| ;
$$

$2^{\circ}$ if $b_{0}>0$, then there is a constant $\tilde{K} \in R_{+}$such that for all $(x, y) \in\left[-b_{0}, 0\right] \times$ $\times R, l \in R, l \neq 0$ we have $l^{-2}[\varphi(x, y+l)-2 \varphi(x, y)+\varphi(x, y-l)] \leqq \widetilde{K}$;

$3^{\circ} f:\left[0, a_{0}\right] \times R \times R \times C(B, R) \times R \rightarrow R$ is of class $C^{2}$;

$4^{\circ}$ there are a constant $N \geqq \tilde{M}$ and a nondecreasing function $V \in C\left([\tilde{M}, N], R_{+}\right)$, $\int_{\tilde{M}}^{N} \mathrm{~d} t / V(t) \geqq a_{0}$, such that for all $t \in R_{+}, \quad(x, y, p, w, q) \in\left[0, a_{0}\right] \times R \times[-t, t] \times$ $\times C(B, R, t) \times R$ we have $|f(x, y, p, w, q)| \leqq V(t)$; 
$5^{\circ}$ there are constants $N_{1} \geqq L, A>0$ and a nondecreasing function $W \in$ $\in C\left(\left[\tilde{L}, N_{1}\right], R_{+}\right), \int_{\tilde{L}}^{N_{1}} \mathrm{~d} t /[(2 t+1) W(N+3 t)] \geqq a_{0}$ such that for all $t \in R_{+}$, $(x, y, p, w, q) \in\left[0, a_{0}\right] \times R \times[-N, N] \times C_{0+L}(B, R, t) \times R$ we have

$$
\begin{array}{ll}
\left|D_{q} f(x, y, p, w, q)\right| \leqq A, & \left|D_{y} f(x, y, p, w, q)\right| \leqq W(t), \\
\left|D_{p} f(x, y, p, w, q)\right| \leqq W(t), & \left\|D_{w} f(x, y, p, w, q)\right\| \leqq W(t) ;
\end{array}
$$

$6^{\circ}$ for all $(x, y, p, w, q) \in\left[0, a_{0}\right] \times R \times[-N, N] \times C_{0+L}\left(B, R, N+3 N_{1}\right) \times$ $\times\left[-N_{1}, N_{1}\right], \bar{w} \in C(B, R), \bar{w} \geqq 0$, we have $D_{w} f(x, y, p, w, q)(\bar{w}) \geqq 0$;

$7^{\circ}$ the derivatives $D_{y y}^{2} f, D_{y p}^{2} f, D_{y w}^{2} f, D_{y q}^{2} f, D_{p p}^{2} f, D_{p w}^{2} f, D_{p q}^{2} f, D_{w w}^{2} f, D_{w q}^{2} f$ are bounded and $D_{q q}^{2} f \leqq 0$ on $\left[0, a_{0}\right] \times R \times[-N, N] \times C_{0+L}\left(B, R, N+3 N_{1}\right) \times$ $\times\left[-N_{1}, N_{1}\right]$;

$8^{\circ}$ if $b_{0}=0$, then there are constants $\delta \in\left(0, a_{0}\right], \mu>0$ such that $D_{q q}^{2} f \leqq-\mu$ on $[0, \delta] \times R \times[-N, N] \times C_{0+L}\left(B, R, N+3 N_{1}\right) \times\left[-N_{1}, N_{1}\right]$.

Let $Z$ be a set of all integers, and let $2 Z$ be a set of all even numbers. Now we introduce a difference scheme for (1), (2). For $h, k>0$ we define $x^{(i)}=i h, i=0,1, \ldots, n_{0}$, $n_{0} h=a_{0}$ and $y^{(j)}=j k, j \in Z$. If $b_{0}>0$, then there is an integer $n_{1}>0$ such that $-n_{1} h \leqq-b_{0}<\left(-n_{1}+1\right) h$. We define $x^{(i)}=i h, i=-n_{1}+1, \ldots,-1$, and $x^{\left(-n_{1}\right)}=-b_{0}$.

Let $U=\{(h, k): A<k / h\}, E^{*}=\left\{\left(x^{(i)}, y^{(j)}\right): i=0, \ldots, n_{0}, j \in Z\right\}$. For $i=$ $=0, \ldots, n_{0}-1, j \in 2 Z$ we write $P_{i j}=\left[x^{(i)}, x^{(i+1)}\right] \times\left[y^{(j-1)}, y^{(j+1)}\right], \quad Q_{i}=$ $=\bigcup_{j \in 2 Z} P_{i j}$. If $v: E^{*} \rightarrow R$, then we denote $v^{(i, j)}=v\left(x^{(i)}, y^{(j)}\right), i=0, \ldots, n_{0}, j \in Z$. Let $\Delta_{0}, \Delta_{1}$ be operators defined by

$$
\Delta_{0} v^{(i, j)}=\frac{1}{h}\left[v^{(i+1, j)}-v^{(i, j)}\right], \quad \Delta_{1} v^{(i, j)}=\frac{1}{2 k}\left[v^{(i, j+1)}-v^{(i, j-1)}\right] .
$$

Furthermore, let $\Delta_{i^{\prime} j^{\prime}}^{2} v^{(i, j)}=\Delta_{i^{\prime}}\left(\Delta_{j^{\prime}}, v^{(i, j)}\right), i^{\prime}, j^{\prime}=0,1$.

Let $\varphi_{h k}:\left[-b_{0}, 0\right] \times R \rightarrow R$ be a function defined in the following way:

(i) If $b_{0}>0$, then for each $(x, y) \in\left[-b_{0}, 0\right] \times R$ there are $i,-n_{1} \leqq i<0$ and $j \in 2 Z$ such that $(x, y) \in\left[x^{(i)}, x^{(i+1)}\right] \times\left[y^{(j-1)}, y^{(j+1)}\right]$. Then we write $\varphi_{h k}(x, y)=$ $=\varphi\left(x^{(i)}, y^{(j-1)}\right)+\left(x-x^{(i)}\right) \Delta_{0} \varphi\left(x^{(i)}, y^{(j-1)}\right)+\left(y-y^{(j-1)}\right) \Delta_{1} \varphi\left(x^{(i)}, y^{(j)}\right)+$ $+\left(x-x^{(i)}\right)\left(y-y^{(j-1)}\right) \Delta_{01}^{2} \varphi\left(x^{(i)}, y^{(j)}\right)$.

(ii) If $b_{0}=0$, then for each $y \in R$ there is $j \in 2 Z$ such that $y \in\left[y^{(j-1)}, y^{(j+1)}\right]$. Then we write $\varphi_{h k}(y)=\varphi\left(y^{(j-1)}\right)+\left(y-y^{(j-1)}\right) \Delta_{1} \varphi\left(y^{(j)}\right)$.

For any $(h, k) \in U$ let us define the function $u_{h k}:\left[-b_{0}, a_{0}\right] \times R \rightarrow R$. We use the mathematical induction in the following way:

(i) Let $v^{(0, j)}=\varphi\left(0, y^{(j)}\right), j \in Z$ and $u_{h k}(x, y)=\varphi_{h k}(x, y)$ for $(x, y) \in\left[-b_{0}, 0\right] \times R$.

(ii) If for some $i, 0 \leqq i \leqq n_{0}-1$ we have defined $v^{(i, j)}, j \in Z$ and $u_{h k}$ on $\left(\left[-b_{0}, 0\right] \times R\right) \cup Q_{0} \cup \ldots \cup Q_{i-1}$, then

$$
\begin{aligned}
& v^{(i+1, j)}=\frac{1}{2}\left(v^{(i, j+1)}+v^{(i, j-1)}\right)+ \\
& +h f\left(x^{(i)}, y^{(j)}, \frac{1}{2}\left(v^{(i, j+1)}+v^{(i, j-1)}\right),\left(u_{h k}\right)_{\left(x^{(i)}, y^{(j)}\right)}, \Delta_{1} v^{(i, j)}\right), \text { where } j \in Z,
\end{aligned}
$$




$$
\begin{aligned}
& u_{h k}(x, y)=v^{(i, j-1)}+\left(x-x^{(i)}\right) \Delta_{0} v^{(i, j-1)}+\left(y-y^{(j-1)}\right) \Delta_{1} v^{(i, j)}+ \\
& +\left(x-x^{(i)}\right)\left(y-y^{(j-1)}\right) \Delta_{01}^{2} v^{(i, j)}, \quad \text { where }(x, y) \in P_{i j}, \quad j \in 2 Z .
\end{aligned}
$$

It is easy to see that (4) defines a continuous function on $\left[-b_{0}, a_{0}\right] \times R$. In the sequel we will write $\left(u_{h k}\right)_{(i, j)}$ instead of $\left(u_{h k}\right)_{\left(x^{(i)}, y^{(j)}\right)}$.

\section{PROPERTIES OF A SOLUTION OF THE DIFFERENCE EQUATION}

Lemma 1. If $f \in C\left(\left[0, a_{0}\right] \times R \times R \times C(B, R) \times R, R\right)$, and conditions $1^{\circ}, 4^{\circ}$ of Assumption $\mathrm{H}$ are satisfied, then for all $i=0, \ldots, n_{0}, j \in Z$ we have

$$
\left|v^{(i, j)}\right| \leqq N .
$$

Proof. It follows from (3) that for $i=0, \ldots, n_{0}-1, j \in Z$ we have

$$
\begin{aligned}
& \left|v^{(i+1, j)}\right| \leqq \frac{1}{2}\left|v^{(i, j+1)}+v^{(i, j-1)}\right|+ \\
& +h\left|f\left(x^{(i)}, y^{(j)}, \frac{1}{2}\left(v^{(i, j+1)}+v^{(i, j-1)}\right),\left(u_{h k}\right)_{(i, j)}, \Delta_{1} v^{(i, j)}\right)\right| .
\end{aligned}
$$

Let $\bar{v}^{(i)}=\sup \left\{\left|v^{(\tau, j)}\right|:-n_{1} \leqq \tau \leqq i, j \in Z\right\}$. The boundness of $\varphi$ implies that $\bar{i}^{(i)}<+\infty$. From the condition $4^{\circ}$ of Assumption $\mathrm{H}$ we have $\bar{v}^{(i+1)} \leqq \bar{v}^{(i)}+$ $+h V\left(\bar{v}^{(i)}\right)$, and hence

$$
\frac{1}{h}\left[\bar{v}^{(i+1)}-\bar{v}^{(i)}\right] \leqq V\left(\bar{v}^{(i)}\right), \quad i=0, \ldots, n_{0}-1 .
$$

Let us consider the Cauchy problem

$$
D_{x} w(x)=V(w(x)), \quad w(0)=\tilde{M} .
$$

If $w$ is a solution of (7), then it is a nondecreasing function and hence $D_{x} w$ is a composition of two nondecreasing functions. Thus $w$ is a convex function and from (6) we see that $\bar{v}^{(i)} \leqq w\left(x^{(i)}\right), i=0, \ldots, n_{0}$. We also have that $w$ satisfies

$$
\int_{\tilde{M}}^{w(x)} \frac{\mathrm{d} t}{V(t)}=x .
$$

From the condition $4^{\circ}$ of Assumption $H$ we have then $w(x) \leqq N, x \in\left[0, a_{0}\right]$. Therefore we have $\bar{v}^{(i)} \leqq N, i=0, \ldots, n_{0}$, which is equivalent to (5).

Lemma 2. If $f:\left[0, a_{0}\right] \times R \times R \times C(B, R) \times R \rightarrow R$ is of class $C^{1},(h, k) \in U$ and conditions $1^{\circ}, 4^{\circ}, 5^{\circ}$ of Assumption $\mathrm{H}$ are satisfied, then for all $i=0, \ldots, n_{0}$, $j \in Z$ we have

$$
\left|\Delta_{1} v^{(i, j)}\right| \leqq N_{1} .
$$

Proof. It follows from (3) that for $i=0, \ldots, n_{0}-1, j \in Z$ we have

$$
\begin{aligned}
& \Delta_{1} v^{(i+1, j)}=\frac{1}{2}\left(\Delta_{1} v^{(i, j+1)}+\Delta_{1} v^{(i, j-1)}\right)+ \\
& +\frac{h}{2 k}\left[f\left(x^{(i)}, y^{(j+1)}, \frac{1}{2}\left(v^{(i, j+2)}+v^{(i, j)}\right),\left(u_{h k}\right)_{(i, j+1)}, \Delta_{1} v^{(i, j+1)}\right)-\right. \\
& \left.-f\left(x^{(i)}, y^{(j-1)}, \frac{1}{2}\left(v^{(i, j)}+v^{(i, j-2)}\right),\left(u_{h k}\right)_{(i, j-1)}, \Delta_{1} v^{(i, j-1)}\right)\right] .
\end{aligned}
$$


Using the Lagrange theorem we obtain

$$
\begin{aligned}
& \Delta_{1} v^{(i+1, j)}=\Delta_{1} v^{(i, j+1)}\left[\frac{1}{2}+\frac{h}{2 k} D_{q} f\left(P^{(i, j)}\right)\right]+ \\
& +\Delta_{1} v^{(i, j-1)}\left[\frac{1}{2}-\frac{h}{2 k} D_{q} f\left(P^{(i, j)}\right)\right]+ \\
& +h D_{y} f\left(P^{(i, j)}\right)+\frac{h}{2}\left(\Delta_{1} v^{(i, j+1)}+\Delta_{1} v^{(i, j-1)}\right) D_{p} f\left(P^{(i, j)}\right)+ \\
& +h D_{w} f\left(P^{(i, j)}\right)\left(r_{i, j}\right),
\end{aligned}
$$

where $P^{(i, j)}$ is an intermediate point, and $r_{i, j}$ is defined by

$$
r_{i, j}=\frac{1}{2 k}\left[\left(u_{h k}\right)_{(i, j+1)}-\left(u_{h k}\right)_{(i, j-1)}\right] .
$$

Let $z^{(i)}=\sup \left\{\left|\Delta_{1} v^{(\tau, j)}\right|:-n_{1} \leqq \tau \leqq i, j \in Z\right\}$. From (5) it follows that $z^{(i)}<+\infty$. Since $\left|D_{q} f\left(P^{(i, j)}\right)\right| \leqq A$ and $(h, k) \in U$, we have

$$
\left|\Delta_{1} v^{(i, j+1)}\left[\frac{1}{2}+\frac{h}{2 k} D_{q} f\left(P^{(i, j)}\right)\right]+\Delta_{1} v^{(i, j-1)}\left[\frac{1}{2}-\frac{h}{2 k} D_{q} f\left(P^{(i, j)}\right)\right]\right| \leqq z^{(i)} .
$$

Thus the above inequality, (5), the condition $5^{\circ}$ of Assumption $\mathrm{H}$ and $\left\|r_{i, j}\right\|_{0} \leqq z^{(l)}$ yield

and hence

$$
z^{(i+1)} \leqq\left(1+2 h W\left(N+3 z^{(i)}\right)\right) z^{(i)}+h W\left(N+3 z^{(i)}\right),
$$

$$
\frac{1}{h}\left[z^{(i+1)}-z^{(i)}\right] \leqq\left(2 z^{(i)}+1\right) W\left(N+3 z^{(i)}\right), \quad i=0, \ldots, n_{0}-1 .
$$

Taking into consideration the Cauchy problem

$$
D_{x} w(x)=(2 w(x)+1) W(N+3 w(x)), \quad w(0)=\tilde{L},
$$

and using the same arguments as in the proof of Lemma 1. we obtain (8).

Lemma 3. Suppose that Assumption $\mathrm{H}$ is satisfied and that $(h, k) \in U$. Then for sufficiently small $k, h$ there is a constant $N_{2} \in R_{+}$such that

$$
\begin{aligned}
& \Delta_{11}^{2} v^{(i, j)} \leqq \frac{N_{2}}{x^{(i)}}, \text { for } \quad b_{0}=0, \quad i=1, \ldots, n_{0}, \quad j \in Z, \\
& \Delta_{11}^{2} v^{(i, j)} \leqq N_{2}, \text { for } b_{0}>0, \quad i=0, \ldots, n_{0}, \quad j \in Z .
\end{aligned}
$$

Proof. We will first prove (9). From (3) it follows that for $i=0, \ldots, n_{0}-1$, $j \in Z$ we have

$$
\begin{aligned}
& \Delta_{11}^{2} v^{(i+1, j)}=\frac{1}{2}\left(\Delta_{11}^{2} v^{(i, j+1)}+\Delta_{11}^{2} v^{(i, j-1)}\right)+ \\
& +\frac{h}{(2 k)^{2}}\left[f\left(x^{(i)}, y^{(j+2)}, \frac{1}{2}\left(v^{(i, j+3)}+v^{(i, j+1)}\right),\left(u_{h k}\right)_{(i, j+2)}, \Delta_{1} v^{(i, j+2)}\right)+\right. \\
& -2 f\left(x^{(i)}, y^{(j)}, \frac{1}{2}\left(v^{(i, j+1)}+v^{(i, j-1)}\right),\left(u_{h k}\right)_{(i, j)}, \Delta_{1} v^{(i, j)}\right)+ \\
& \left.+f\left(x^{(i)}, y^{(j-2)}, \frac{1}{2}\left(v^{(i, j-1)}+v^{(i, j-3)}\right),\left(u_{h k}\right)_{(i, j-2)}, \Delta_{1} v^{(i, j-2)}\right)\right] .
\end{aligned}
$$


Let $r_{i, j}$ have the same meaning as in the proof of Lemma 2 and let

$$
\begin{aligned}
& q_{i, j}=\frac{1}{(2 k)^{2}}\left[\left(u_{h k}\right)_{(i, j+2)}-2\left(u_{h k}\right)_{(i, j)}+\left(u_{h k}\right)_{(i, j-2)}\right], \\
& Q^{(i, j)}=\left(x^{(i)}, y^{(j)}, \frac{1}{2}\left(v^{(i, j+1)}+v^{(i, j-1)}\right),\left(u_{h k}\right)_{(i, j)}, \Delta_{1} v^{(i, j)}\right) .
\end{aligned}
$$

From the relations

$$
\begin{aligned}
& v^{\left(i, j^{\prime}+1\right)}+v^{\left(i, j^{\prime}-1\right)}-\left(v^{\left(i, j^{\prime}-1\right)}+v^{\left(i, j^{\prime}-3\right)}\right)=2 k\left(\Delta_{1} v^{\left(i, j^{\prime}\right)}+\Delta_{1} v^{\left(i, j^{\prime}-2\right)}\right), \\
& \Delta_{1} v^{\left(i, j^{\prime}+1\right)}-\Delta_{1} v^{\left(i, j^{\prime}-1\right)}=2 k \Delta_{11}^{2} v^{\left(i, j^{\prime}\right)},
\end{aligned}
$$

and from Taylor's formula we obtain

$$
\begin{aligned}
& \Delta_{11}^{2} v^{(i+1, j)}=\Delta_{11}^{2} v^{(i, j+1)}\left[\frac{1}{2}+\frac{h}{2 k} D_{q} f\left(Q^{(i, j)}\right)+h D_{y q}^{2} f\left(Q_{1}^{(i, j)}\right)+\right. \\
& \left.+\frac{h}{2}\left(\Delta_{1} v^{(i, j+2)}+\Delta_{1} v^{(i, j)}\right) D_{q p}^{2} f\left(Q_{1}^{(i, j)}\right)+h D_{q w}^{2} f\left(Q_{1}^{(i, j)}\right)\left(r_{i, j+1}\right)\right]+ \\
& +\Delta_{11}^{2} v^{(i, j-1)}\left[\frac{1}{2}-\frac{h}{2 k} D_{q} f\left(Q^{(i, j)}\right)+h D_{y q}^{2} f\left(Q_{2}^{(i, j)}\right)+\right. \\
& \left.+\frac{h}{2}\left(\Delta_{1} v^{(i, j)}+\Delta_{1} v^{(i, j-2)}\right) D_{q p}^{2} f\left(Q_{2}^{(i, j)}\right)+h D_{q w}^{2} f\left(Q_{2}^{(i, j)}\right)\left(r_{i, j-1}\right)\right]+ \\
& +\frac{h}{2}\left(\Delta_{11}^{2} v^{(i, j+1)}+\Delta_{11}^{2} v^{(i, j-1)}\right) D_{p} f\left(Q^{(i, j)}\right)+h D_{w} f\left(Q^{(i, j)}\right)\left(q_{i, j}\right)+R^{(i, j)},
\end{aligned}
$$

where $Q_{1}^{(i, j)}, Q_{2}^{(i, j)}$ are intermediate points and

$$
\begin{aligned}
& R^{(i, j)}=\frac{h}{2}\left[D_{y y}^{2} f\left(Q_{1}^{(i, j)}\right)+D_{y y}^{2} f\left(Q_{2}^{(i, j)}\right)\right]+ \\
& +\frac{h}{2}\left[\frac{1}{4}\left(\Delta_{1} v^{(i, j+2)}+\Delta_{1} v^{(i, j)}\right)^{2} D_{p p}^{2} f\left(Q_{1}^{(i, j)}\right)+\right. \\
& \left.+\left(\Delta_{1} v^{(i, j+2)}+\Delta_{1} v^{(i, j)}\right) D_{y p}^{2} f\left(Q_{1}^{(i, j)}\right)\right]+ \\
& +\frac{h}{2}\left[\frac{1}{4}\left(\Delta_{1} v^{(i, j)}+\Delta_{1} v^{(i, j-2)}\right)^{2} D_{p p}^{2} f\left(Q_{2}^{(i, j)}\right)+\right. \\
& \left.+\left(\Delta_{1} v^{(i, j)}+\Delta_{1} v^{(i, j-2)}\right) D_{y p}^{2} f\left(Q_{2}^{(i, j)}\right)\right]+ \\
& +h\left[\frac{1}{2} D_{w w}^{2} f\left(Q_{1}^{(i, j)}\right)\left(r_{i, j+1}\right)+D_{y w}^{2} f\left(Q_{1}^{(i, j)}\right)+\right. \\
& \left.+\frac{1}{2}\left(\Delta_{1} v^{(i, j)}+\Delta_{1} v^{(i, j+2)}\right) D_{p w}^{2} f\left(Q_{1}^{(i, j)}\right)\right]\left(r_{i, j+1}\right)+ \\
& +h\left[\frac{1}{2} D_{w w}^{2} f\left(Q_{2}^{(i, j)}\right)\left(r_{i, j-1}\right)+D_{y w}^{2} f\left(Q_{2}^{(i, j)}\right)+\right. \\
& \left.+\frac{1}{2}\left(\Delta_{1} v^{(i, j)}+\Delta_{1} v^{(i, j-2)}\right) D_{p w}^{2} f\left(Q_{2}^{(i, j)}\right)\right]\left(r_{i, j-1}\right)+ \\
& +\frac{h}{2}\left[\Delta_{11}^{2} v^{(i, j+1)}\right]^{2} D_{q q}^{2} f\left(Q_{1}^{(i, j)}\right)+\frac{h}{2}\left[\Delta_{11}^{2} v^{(i, j-1)}\right]^{2} D_{q q}^{2} f\left(Q_{2}^{(i, j)}\right) .
\end{aligned}
$$


We will first estimate $\Delta_{11}^{2} v^{(i, j)}$ for $i$ such that $x^{(i)} \leqq \delta$, where $\delta$ is the constant from the condition $8^{\circ}$ from Assumption $\mathrm{H}$. For $i=0, \ldots, n_{0}-1, j \in Z$ we write

$$
\begin{aligned}
& A_{1}^{(i, j)}=D_{y q}^{2} f\left(Q_{1}^{(i, j)}\right)+\frac{1}{2}\left(\Delta_{1} v^{(i, j+2)}+\Delta_{1} v^{(i, j)}\right) D_{q p}^{2} f\left(Q_{1}^{(i, j)}\right)+ \\
& +D_{q w}^{2} f\left(Q_{1}^{(i, j)}\right)\left(r_{i, j+1}\right)+\frac{1}{2} D_{p} f\left(Q_{1}^{(i, j)}\right), \\
& A_{2}^{(i, j)}=D_{y q}^{2} f\left(Q_{2}^{(i, j)}\right)+\frac{1}{2}\left(\Delta_{1} v^{(i, j)}+\Delta_{1} v^{(i, j-2)}\right) D_{q p}^{2} f\left(Q_{2}^{(i, j)}\right)+ \\
& +D_{q w}^{2} f\left(Q_{2}^{(i, j)}\right)\left(r_{i, j-1}\right)+\frac{1}{2} D_{p} f\left(Q_{2}^{(i, j)}\right) .
\end{aligned}
$$

There are constants $d_{1}, d_{2} \in R_{+}, d_{1}>d_{2}$, and

$$
c=\min \left(\frac{\mu}{2}, A \frac{1+d_{1} h}{2 N_{1}}\right)
$$

such that

$$
A_{1}^{(i, j)}+A_{2}^{(i, j)} \leqq d_{1}, \quad R^{(i, j)} \leqq d_{2} h-c\left[\Delta_{11}^{2} v^{(i, j+1)}+\Delta_{11}^{2} v^{(i, j-1)}\right] h .
$$

Let $h, k$ be sufficiently small so that

$$
\frac{1}{2}+\frac{h}{2 k} D_{q} f\left(Q^{(i, j)}\right)+h A_{1}^{(i, j)} \geqq 0, \quad \frac{1}{2}-\frac{h}{2 k} D_{q} f\left(Q^{(i, j)}\right)+h A_{2}^{(i, j)} \geqq 0 .
$$

We introduce the following notations

$$
\begin{aligned}
& S^{(i, j)}=\max \left\{\Delta_{11}^{2} v^{(i, j+1)}, \Delta_{11}^{2} v^{(i, j-1)}, 0\right\}, \\
& \widetilde{S}^{(i)}=\sup \left\{S^{(\tau, j)}: 0 \leqq \tau \leqq i, j \in Z\right\} .
\end{aligned}
$$

From (5) it follows that $\widetilde{S}^{(i)}<+\infty$. Therefore we have

$$
\begin{aligned}
& \Delta_{11}^{2} v^{(i+1, j)} \leqq S^{(i, j)}\left[1+d_{1} h\right]+ \\
& +W\left(N+3 N_{1}\right) \tilde{S}^{(i)} h+d_{2} h-c\left(S^{(i, j)}\right)^{2} h .
\end{aligned}
$$

From the inequality

$$
\Delta_{11}^{2} v^{(i, j)}=\frac{1}{2 k}\left(\Delta_{1} v^{(i, j+1)}-\Delta_{1} v^{(i, j-1)}\right) \leqq \frac{N_{1}}{k},
$$

and from conditions $A<k / h$ and $c \leqq A\left(1+d_{1} h\right) / 2 N_{1}$ we obtain

$$
\Delta_{11}^{2} v^{(i, j)} \leqq \frac{1+d_{1} h}{2 c h} .
$$

Let us consider the polynomial $H(y)=y\left(1+d_{1} h\right)+W\left(N+3 N_{1}\right) \tilde{S}^{(i)} h+d_{2} h-$ - chy $y^{2}$. It is easy to see that $D_{y} H(y)=1+d_{1} h-2 c h y \geqq 0$ for $y \leqq\left(1+d_{1} h\right) / 2 c h$. By force of (11) we derive then

$$
\Delta_{11}^{2} v^{(i+1, j)} \leqq \widetilde{S}^{(i)}\left[1+\left(d_{1}+W\left(N+3 N_{1}\right)\right) h\right]+d_{2} h-c\left(\widetilde{S}^{(i)}\right)^{2} h .
$$

The right hand side of the above inequality is positive, which gives

$$
\tilde{S}^{(i+1)} \leqq \widetilde{S}^{(i)}\left[1+\left(d_{1}+W\left(N+3 N_{1}\right)\right) h\right]+d_{2} h-c\left(\widetilde{S}^{(i)}\right)^{2} h .
$$


Hence for $\hat{S}^{(i)}=\widetilde{S}^{(i)}+1$ we have

$$
\begin{aligned}
& \hat{S}^{(i+1)} \leqq \hat{S}^{(i)}\left[1+\left(d_{1}+W\left(N+3 N_{1}\right)+2 c\right) h\right]+ \\
& +d_{2} h-\left(d_{1}+W\left(N+3 N_{1}\right)+c\right) h-c\left(S^{(i)}\right)^{2} h .
\end{aligned}
$$

Using the condition $d_{1}>d_{2}$ and putting $\tilde{d}=d_{1}+W\left(N+3 N_{1}\right)+2 c$ we obtain

$$
\hat{S}^{(i+1)} \leqq \hat{S}^{(i)}(1+\tilde{d} h)-\operatorname{ch}\left(\hat{S}^{(i)}\right)^{2} \text {. }
$$

Let $h$ be so small that $\tilde{d} h \leqq \frac{1}{2}$. Then multiplying the last inequality by $(1-\tilde{d} h)^{i+1}$ and putting $W^{(i)}=(1-\tilde{d} h)^{i} \hat{S}^{(i)}$ we get

$$
\begin{aligned}
& W^{(i+1)} \leqq W^{(i)}-\operatorname{ch}\left(W^{(i)}\right)^{2}(1-\tilde{d} h)^{-i+1} \leqq W^{(i)}-\operatorname{ch}\left(W^{(i)}\right)^{2}, \quad i>0, \\
& W^{(1)} \leqq W^{(0)}-\operatorname{ch}\left(W^{(0)}\right)^{2}(1-\tilde{d} h) \leqq W^{(0)}-\frac{c}{2}\left(W^{(0)}\right)^{2},
\end{aligned}
$$

and hence

$$
\frac{1}{h}\left[W^{(i+1)}-W^{(i)}\right] \leqq-\frac{c}{2}\left(W^{(i)}\right)^{2} .
$$

Let us consider the following Cauchy problem

$$
D_{x} w(x)=-\frac{c}{2} w^{2}(x), \quad w(0)=W^{(0)} .
$$

The solution of this problem is given by

$$
w(x)=\frac{1}{\frac{c}{2} x+\frac{1}{W^{(0)}}} .
$$

We see that $w$ is convex and then by force of $(12)$ we have

$$
W^{(i)} \leqq w\left(x^{(i)}\right)=\frac{1}{\frac{c}{2} x^{(i)}+\frac{1}{W^{(0)}}}<\frac{2}{c x^{(i)}}, \quad i>0 .
$$

From the above inequality we derive

$$
(1-\tilde{d} h)^{i}\left(\tilde{S}^{(i)}+1\right) \leqq \frac{2}{c x^{(i)}},
$$

and hence from the condition $\tilde{d} h \leqq \frac{1}{2}$ we have

$$
\tilde{S}^{(i)} \leqq \frac{1}{x^{(i)}}\left[\frac{2}{c}(1-\tilde{d} h)^{-i}-i h\right] \leqq \frac{1}{x^{(i)}}\left\{\frac{2}{c}\left[(1-\tilde{d} h)^{-1 / d h}\right]^{d i h}-i h\right\} \leqq \frac{\tilde{N}_{2}}{x^{(i)}},
$$

where

$$
\tilde{N}_{2}=\frac{2}{c} \exp (\tilde{d} \delta \ln 4)-\delta
$$


For $i$ such that $x^{(i)}>\delta$ we analogously derive

$$
\tilde{S}^{(i+1)} \leqq \widetilde{S}^{(i)}\left[1+\left(d_{1}+W\left(N+3 N_{1}\right)\right) h\right]+d_{2} h .
$$

By force of the mathematical induction we have

$$
\tilde{S}^{(i)} \leqq \exp \left(d a_{0}\right) \tilde{S}^{([\delta / h])}+\frac{d_{2}}{d}\left[\exp \left(d a_{0}\right)-1\right],
$$

where $\hat{d}=d_{1}+W\left(N+3 N_{1}\right)$ and $[t]$ denotes the integral part of $t$. Supposing that $h$ is so small that $h \leqq \delta / 2$ and putting

$$
N_{2}=\max \left\{\tilde{N}_{2}, \frac{2 \tilde{N_{2}}}{\delta} \exp \left(d a_{0}\right)+\frac{d_{2}}{d}\left[\exp \left(d a_{0}\right)-1\right] \text { we get }(9)\right. \text {. }
$$

In order to prove (10), let us adopt the previous notations $r_{i, j}, q_{i, j}, Q^{(i, j)}, Q_{1}^{(i, j)}$, $Q_{2}^{(i, j)}, R^{(i, j)}, A_{1}^{(i, j)}, A_{2}^{(i, J)}$ and let $h, k$ be so small that

$$
\frac{1}{2}+\frac{h}{2 k} D_{q} f\left(Q^{(i, j)}\right)+h A_{1}^{(i, j)} \geqq 0, \frac{1}{2}-\frac{h}{2 k} D_{q} f\left(Q^{(i, j)}\right)+h A_{2}^{(i, j)} \geqq 0 .
$$

There are constants $d_{1}, d_{2}$ such that

$$
A_{1}^{(i, j)}+A_{2}^{(i, j)} \leqq d_{1}, \quad R^{(i, j)} \leqq d_{2} h .
$$

Using the previous arguments we prove that

$$
\widetilde{S}^{(i+1)} \leqq \widetilde{S}^{(i)}\left[1+\left(d_{1}+W\left(N+3 N_{1}\right)\right) h\right]+d_{2} h, \quad i=0, \ldots, n_{0}-1,
$$

where $\widetilde{S}^{(i)}=\sup \left\{\Delta_{11}^{2} v^{(\tau, j)}:-n_{1} \leqq \tau \leqq i, j \in Z\right\}$. Now, analogously like (13) we obtain (10) with

$$
N_{2}=\left(\tilde{K}+\frac{d_{2}}{d}\right) \exp \left(d a_{0}\right)-\frac{d_{2}}{\hat{d}},
$$

where $\widetilde{K}$ is the constant from the condition $2^{\circ}$ of Assumption H. This ends the proof.

Let $B$ be some constant such that $B>A$. By $\tilde{U}$ we define the set $\{(h, k): A<$ $<k / h \leqq B\}$.

Lemma 4. Suppose that Assumption $\mathrm{H}$ is satisfied, $(h, k) \in \tilde{U}, \alpha, Y>0,0 \leqq i \leqq n_{0}$ and additionally $i>\alpha / h$ in the case $b_{0}=0$. Then there is a constant $C=C(Y, \alpha)$ for $b_{0}=0$, or $C=C(Y)$ for $b_{0}>0$ such that

$$
\sum_{|j| \leqq Y / k} 2 k\left|\Delta_{11}^{2} v^{(i, j)}\right| \leqq C .
$$

Proof. Let $b_{0}=0, \bar{A} \geqq N_{2} / \alpha$ and $u^{(i, j)}=\Delta_{1} v^{(i, j)}-\bar{A} j k$. Using (9) for any $i>\alpha / h, j \in Z$ we have

$$
\begin{aligned}
& u^{(i, j+1)}-u^{(i, j-1)}=\Delta_{1} v^{(i, j+1)}-\Delta_{1} v^{(i, j-1)}-2 \bar{A} k= \\
& =2 k\left(\Delta_{11}^{2} v^{(i, j)}-\bar{A}\right) \leqq 2 k\left(\frac{N_{2}}{i h}-\bar{A}\right) \leqq 0 .
\end{aligned}
$$


If $b_{0}>0$, then we take $\bar{A} \geqq N_{2}$ and by force of (10) we obtain the same estimate for $i=0, \ldots, n_{0}, j \in Z$. From this we have

$$
\begin{aligned}
& \sum_{|j| \leqq Y / k} 2 k\left|\Delta_{1} u^{(i, j)}\right|=\sum_{|j| \leqq Y / k}\left[u^{(i, j+1)}-u^{(i, j-1)}\right] \leqq \\
& \leqq 4 \max \left|u^{(i, j)}\right| \leqq 4\left(N_{1}+\bar{A} Y\right),
\end{aligned}
$$

and

$$
\begin{aligned}
& \sum_{|j| \leqq Y / k} 2 k\left|\Delta_{11}^{2} v^{(i, j)}\right|=\sum_{|j| \leqq Y / k}\left|\Delta_{1} v^{(i, j+1)}-\Delta_{1} v^{(i, j-1)}\right|= \\
& =\sum_{|j| \leqq Y / k}\left|u^{(i, j+1)}-u^{(i, j-1)}+2 \bar{A} k\right| \leqq \sum_{|j| \leqq Y / k} 2 k\left|\Delta_{1} u^{(i, j)}\right|+ \\
& +4 \bar{A} Y+2 \bar{A} B a_{0} .
\end{aligned}
$$

Thus (14) is satisfied with $C=4 N_{1}+8 \bar{A} Y+2 \bar{A} B a_{0}$.

Remark 2. The analogous properties to that proved in Lemmas 2-4 we may obtain also for the operator $\tilde{\Delta}_{1}$ defined by $\widetilde{\Delta}_{1} v^{(i, j)}=(1 / k)\left[v^{(i, j)}-v^{(i, j-1)}\right]$.

For any $1 \leqq i \leqq n_{0}, j \in Z, 0 \leqq n \leqq i-1$ let $U_{i j}(n)=\{s \in Z: s-j+i-$ $-n \in 2 Z\}$.

Lemma 5. If $f:\left[0, a_{0}\right] \times R \times R \times C(B, R) \times R \rightarrow R$ is of class $C^{1},(h, k) \in U$ and conditions $1^{\circ}, 4^{\circ}, 5^{\circ}$ of Assumption $\mathrm{H}$ are satisfied, then there are constants $a_{i, j}^{n, s} \geqq 0, \eta_{i, j}^{n}, i=1, \ldots, n_{0}, j \in Z, n=0, \ldots, i-1, s \in U_{i j}(n), j-(i-n) \leqq s \leqq$ $\leqq j+(i-n)$, such that

$$
\begin{aligned}
& \Delta_{1} v^{(i, j)}=\sum_{\substack{s=j-(i-n) \\
s \in U_{i j}(n)}}^{j+(i-n)} a_{i, j}^{n, s} \Delta_{1} v^{(n, s)}+\eta_{i, j}^{n}, \sum_{\substack{s=j-(i-n) \\
s \in U_{i j}(n)}}^{j+(i-n)} a_{i, j}^{n, s}=1, \\
& \left|\eta_{i, j}^{n}\right| \leqq(i-n) h c_{1},
\end{aligned}
$$

where $c_{1}=W\left(N+3 N_{1}\right)\left(1+2 N_{1}\right)$.

Proof. Analogously like in Lemma 2 we get

$$
\begin{aligned}
& \Delta_{1} v^{(i, j)}=\Delta_{1} v^{(i-1, j+1)}\left[\frac{1}{2}+\frac{h}{2 k} D_{q} f\left(P^{(i-1, j)}\right)\right]+ \\
& +\Delta_{1} v^{(i-1, j-1)}\left[\frac{1}{2}-\frac{h}{2 k} D_{q} f\left(P^{(i-1, j)}\right)\right]+\eta_{i, j}^{i-1},
\end{aligned}
$$

where

$$
\begin{aligned}
& \eta_{i, j}^{i-1}=h D_{y} f\left(P^{(i-1, j)}\right)+ \\
& +\frac{h}{2}\left(\Delta_{1} v^{(i-1, j+1)}+\Delta_{1} v^{(i-1, j-1)}\right) D_{p} f\left(P^{(i-1, j)}\right)+ \\
& +h D_{w} f\left(P^{(i-1, j)}\right)\left(r_{i-1, j}\right), \quad\left|\eta_{i, j}^{i-1}\right| \leqq h c_{1} .
\end{aligned}
$$

Thus the lemma holds for $n=i-1$. Assume that the lemma holds for some $n<i$, 
we will prove it for $n-1$. For $i=1, \ldots, n_{0}, j \in Z$ we have

$$
\begin{aligned}
& \Delta_{1} v^{(i, j)}=\sum_{\substack{s=j-(i-n) \\
s \in U_{i j}(n)}}^{j+(i-n)} a_{i, j}^{n, s} \Delta_{1} v^{(n, s)}+\eta_{i, j}^{n}= \\
& =\sum_{\substack{s=j-(i-n) \\
s \in U_{i j(n)}}}^{j+(i-n)} a_{i, j}^{n, s}\left\{\Delta_{1} v^{(n-1, s+1)}\left[\frac{1}{2}+\frac{h}{2 k} D_{q} f\left(P^{(n-1, s)}\right)\right]+\right. \\
& \left.+\Delta_{1} v^{(n-1, s-1)}\left[\frac{1}{2}-\frac{h}{2 k} D_{q} f\left(P^{(n-1, s)}\right)\right]+\tilde{\eta}_{i, j}^{s}\right\}+\eta_{i, j}^{n},
\end{aligned}
$$

where

$$
\begin{aligned}
& \tilde{\eta}_{i, j}^{\mathrm{s}}=h D_{y} f\left(P^{(n-1, s)}\right)+\frac{h}{2}\left(\Delta_{1} v^{(n-1, s+1)}+\Delta_{1} v^{(n-1, s-1)}\right) D_{p} f\left(P^{(n-1, s)}\right)+ \\
& +h D_{w} f\left(P^{(n-1, s)}\right)\left(r_{n-1, s}\right) .
\end{aligned}
$$

We define constants $a_{i, j}^{n-1, s}, \eta_{i, j}^{n-1}$ in the following way:

$$
\begin{aligned}
& a_{i, j}^{n-1, s}=a_{i, j}^{n, s-1}\left[\frac{1}{2}+\frac{h}{2 k} D_{q} f\left(P^{(n-1, s-1)}\right)\right]+ \\
& +a_{i, j}^{n, s+1}\left[\frac{1}{2}-\frac{h}{2 k} D_{q} f\left(P^{(n-1, s+1)}\right)\right], \\
& s=j-(i-n)+1, \ldots, j+(i-n)-1, \\
& a_{i, j}^{n-1, j-(i-n)-1}=a_{i, j}^{n, j-(i-n)}\left[\frac{1}{2}-\frac{h}{2 k} D_{q} f\left(P^{(n-1, j-(i-n))}\right)\right], \\
& a_{i, j}^{n-1, j+(i-n)+1}=a_{i, j}^{n, j+(i-n)}\left[\frac{1}{2}+\frac{h}{2 k} D_{q} f\left(P^{(n-1, j+(i-n))}\right)\right], \\
& \eta_{i, j}^{n-1}=\sum_{\substack{j=j-(i-n) \\
s \in U_{i j}(n)}}^{j+(i-n)} a_{i, j}^{n, s} \tilde{\eta}_{i, j}^{s}+\eta_{i, j}^{n} .
\end{aligned}
$$

For these constants we have

$$
\begin{aligned}
& \Delta_{1} v^{(i, j)}=\sum_{\substack{s=j-(i-n)-1 \\
s \in U_{i j(n-1)}}}^{j+(i-n)+1} a_{i, j}^{n-1, s} \Delta_{1} v^{(n-1, s)}+\eta_{i, j}^{n-1}, \\
& \sum_{\substack{s=j-(i-n)-1 \\
s \in U_{i j}(n-1)}}^{j+(i-n)+1} a_{i, j}^{n-1, s}=1, \\
& \left|\eta_{i, j}^{n-1}\right| \leqq(i-n+1) h c_{1},
\end{aligned}
$$

which completes the proof of Lemma 5.

Lemma 6. Suppose that Assumption $\mathrm{H}$ is satisfied, $(h, k) \in \tilde{U}, \alpha, Y>0,1 \leqq i \leqq$ $\leqq n_{0}, 0 \leqq n \leqq i-1$ and additionally $i>\alpha / h, n>\alpha / h$ in the case $b_{0}=0$. Then 
there is a constant $L=L(Y, \alpha)$ for $b_{0}=0$, or $L=L(Y)$ for $b_{0}>0$ such that

$$
\sum_{|j| \leqq Y / k} 2 k\left|\Delta_{1} v^{(i, j)}-\Delta_{1} v^{(n, j)}\right| \leqq L h(i-n) .
$$

Proof. We will first prove (15) for the case $i-n \in 2 Z$. By force of Lemma 5 we have

$$
\begin{aligned}
& \sum_{|j| \leqq Y / k} 2 k\left|\Delta_{1} v^{(i, j)}-\Delta_{1} v^{(n, j)}\right| \leqq \\
& \leqq \sum_{|j| \leqq Y / k} \sum_{\substack{s=j-(i-n) \\
s \in U_{i j}(n)}}^{j+(i-n)} a_{i, j}^{n, s}\left|\Delta_{1} v^{(n, s)}-\Delta_{1} v^{(n, j)}\right| 2 k+ \\
& +\left(4 Y+2 B a_{0}\right) c_{1}(i-n) h \leqq \\
& \sum_{|j| \leqq Y / k} \sum_{\substack{s=j-(i-n) \\
s \in U_{i j}(n)}}^{j+(i-n)} a_{i, j}^{n, s} \sum_{r=j-(i-n)}^{j+(i-n)}\left|\Delta_{1} v^{(n, r+1)}-\Delta_{1} v^{(n, r-1)}\right| 2 k+ \\
& +\left(4 Y+2 B a_{0}\right) c_{1}(i-n) h \leqq \\
& \leqq[2(i-n)+1] 2 k \sum_{|r| \leqq Y / k+(i-n)}\left|\Delta_{1} v^{(n, r+1)}-\Delta_{1} v^{(n, r-1)}\right|+ \\
& +\left(4 Y+2 B a_{0}\right) c_{1}(i-n) h .
\end{aligned}
$$

Using (14) with $C=C\left(Y+B a_{0}, \alpha\right)$ for $b_{0}=0$, or $C=C\left(Y+B a_{0}\right)$ for $b_{0}>0$ we get (15) with the constant $L=6 B C+4 Y c_{1}+2 B a_{0} c_{1}$. From Remark 2 we obtain (15) for $i-n \notin 2 Z$.

\section{THE SEQUENCE OF APPROXIMATE SOLUTIONS}

Lemma 7. Suppose that $f:\left[0, a_{0}\right] \times R \times R \times C(B, R) \times R \rightarrow R$ is of class $C^{1}$ and that conditions $1^{\circ}, 4^{\circ}, 5^{\circ}$ of Assumption $\mathrm{H}$ are satisfied. Then there is a sequence $\left\{\left(h_{v}, k_{v}\right)\right\}_{v=1}^{\infty},\left(h_{v}, k_{v}\right) \in \tilde{U}, \lim _{v \rightarrow \infty} h_{v}=\lim _{v \rightarrow \infty} k_{v}=0$, and a function $\tilde{u} \in C\left(\left[0, a_{0}\right] \times R, R\right)$ such that $\lim _{v \rightarrow \infty} u_{h_{v} k_{v}}(x, y)=\tilde{u}(x, y)$ almost uniformly on $\left[0, a_{0}\right] \times R$.

This lemma follows from Lemmas 1, 2 and from Remark 2 .

Let us define sequences $\left\{u^{(v)}\right\}_{v=1}^{\infty},\left\{V^{(v)}\right\}_{v=1}^{\infty},\left\{W^{(v)}\right\}_{v=1}^{\infty}$. We put $u^{(v)}=u_{h_{v} k_{v}}$. If $(x, y) \in\left[0, a_{0}\right] \times R$, then there are $i, j, 0 \leqq i \leqq n_{0}-1, j \in 2 Z$ such that $(x, y) \in$ $\in\left[x^{(i)}, x^{(i+1)}\right) \times\left[y^{(j-1)}, y^{(j+1)}\right)$. Let

$$
\begin{aligned}
& V^{(v)}(x, y)=\Delta_{1} v^{(i, j)}+\left(x-x^{(i)}\right) \Delta_{01}^{2} v^{(i, j)}, \\
& W^{(v)}(x, y)=\Delta_{0} v^{(i, j-1)}+\left(y-y^{(j-1)}\right) \Delta_{01}^{2} v^{(i, j)},
\end{aligned}
$$

where the difference operators are defined for $h=h_{v}, k=k_{v}$. If $i=n_{0}-1$, then we replace in the above definitions the interval $\left[x^{(i)}, x^{(i+1)}\right)$ by $\left[x^{(i)}, x^{(i+1)}\right]$. Thus we have $V^{(v)}, W^{(v)}:\left[0, a_{0}\right] \times R \rightarrow R$ and $V^{(v)}(x, y)=D_{y} u^{(v)}(x, y), W^{(v)}(x, y)=$ $=D_{x} u^{(v)}(x, y)$ a.e. on $\left[0, a_{0}\right] \times R$. 
By $\operatorname{Var}_{Y}\left[V^{(v)}(x, \cdot)\right]$ we denote the variation of the function $V^{(v)}(x, \cdot)$ on $[-Y, Y]$, $Y>0$.

Lemma 8. If Assumption $\mathrm{H}$ is satisfied and $\alpha, Y>0$, then for each $x \in\left(\alpha, a_{0}\right]$, $\left(x \in\left[0, a_{0}\right]\right.$ in the case $\left.b_{0}>0\right)$ and for any integer $v$ we have $\operatorname{Var}_{Y}\left[V^{(v)}(x, \cdot)\right] \leqq 3 C$, where $C$ is the constant from (14).

Proof. For any $x \in\left[0, a_{0}\right]$ there is $i, 0 \leqq i \leqq n_{0}-1$ such that $x \in\left[x^{(i)}, x^{(i+1)}\right)$. Since $V^{(v)}(x, \cdot)$ is a constant function on intervals $\left[y^{(j-1)}, y^{(j+1)}\right), j \in 2 Z$, we have

$$
\begin{aligned}
& \operatorname{Var}_{Y}\left[V^{(v)}(x, \cdot)\right]=\sum_{j \in 2 Z,\left|j k_{v}\right| \leqq Y}\left|V^{(v)}\left(x, y^{(j+1)}\right)-V^{(v)}\left(x, y^{(j-1)}\right)\right| \leqq \\
& \leqq \\
& \sum_{|j| \leqq Y / k_{v}} \mid \Delta_{1} v^{(i, j+1)}+\left(x-x^{(i)}\right) \Delta_{01}^{2} v^{(i, j+1)}-\Delta_{1} v^{(i, j-1)}- \\
& -\left(x-x^{(i)}\right) \Delta_{01}^{2} v^{(i, j-1)} \mid \leqq \sum_{|j| \leqq Y / k_{v}}\left[\left|\Delta_{1} v^{(i, j+1)}-\Delta_{1} v^{(i, j-1)}\right|+\right. \\
& \left.+\frac{x-x^{(i)}}{h_{v}}\left|\Delta_{1} v^{(i+1, j+1)}-\Delta_{1} v^{(i, j+1)}-\Delta_{1} v^{(i+1, j-1)}+\Delta_{1} v^{(i, j-1)}\right|\right] \leqq \\
& \left.\leqq \sum_{|j| \leqq Y / k_{v}} 2 k_{v}\left|\Delta_{11}^{2} v^{(i, j)}\right|+\frac{x-x^{(i)}}{h_{v}}\left(2 k_{v}\left|\Delta_{11}^{2} v^{(i+1, j)}\right|+2 k_{v}\left|\Delta_{11}^{2} v^{(i, j)}\right|\right)\right] .
\end{aligned}
$$

The above inequality and (14) complete the proof of Lemma 8.

By $L(Y)$ we denote a set of all Lebesgue integrable functions $\psi:\left[0, a_{0}\right] \times$ $\times[-Y, Y] \rightarrow R$ with the norm $\|\psi\|_{L_{(Y)}}=\int_{0}^{a_{0}} \int_{-Y}^{Y}|\psi(x, y)| \mathrm{d} x \mathrm{~d} y$.

Lemma 9. If Assumption $\mathrm{H}$ is satisfied, then there is a sequence $\left\{v_{s}\right\}_{s=1}^{\infty}$ and a measurable function $\bar{v}:\left[0, a_{0}\right] \times R \rightarrow R$ such that $\lim _{s \rightarrow \infty}\left\|V^{\left(v_{s}\right)}-\bar{v}\right\|_{L(Y)}=0$.

Proof. Let $Y>0, \alpha \in\left(0, a_{0}\right)$ and let $\left\{x_{r}\right\}_{r=1}^{\infty}$ be a sequence of all rational numbers from the interval $\left[\alpha, a_{0}\right]$. It is easy to see that for any integer $v$ we have $\left|V^{(v)}(x, y)\right| \leqq$ $\leqq 3 N_{1},(x, y) \in\left[0, a_{0}\right] \times R$. From this and from Lemma 8 it follows that assumptions of Helly's theorem are satisfied. Hence for any $x \in\left[\alpha, a_{0}\right]$ there is a subsequence of the sequence $\left\{V^{(v)}(x, \cdot)\right\}_{v=1}^{\infty}$ which is convergent on $[-Y, Y]$. If we apply the diagonal process, then we obtain a subsequence of $\left\{V^{(v)}\right\}_{v=1}^{\infty}$, which is convergent on the set $\left\{(x, y) \in\left[\alpha, a_{0}\right] \times R: x=x_{r}\right.$ for some $\left.r\right\}$. We denote this sequence by $\left\{V^{(v)}\right\}_{v=1}^{\infty}$ again.

We will prove that

$$
\lim _{v, s \rightarrow \infty} \int_{-Y}^{Y}\left|V^{(s)}(x, y)-V^{(v)}(x, y)\right| \mathrm{d} y=0,
$$

uniformly with respect to $x \in\left[\alpha, a_{0}\right]$.

For each $\varepsilon>0$ there is a finite subset $\left\{x_{r_{1}}, \ldots, x_{r_{m}}\right\}$ of the sequence $\left\{x_{r}\right\}_{r=1}^{\infty}$ such that the distance between any two successive elements of this set is less then $\varepsilon / 5 L$, where $L$ is the constant from inequality (15). For sufficiently large $v, s$ we have

$$
\int_{-\boldsymbol{Y}}^{Y}\left|V^{(v)}\left(x_{\boldsymbol{r} l}, y\right)-V^{(s)}\left(x_{\boldsymbol{r} l}, y\right)\right| \mathrm{d} y<\varepsilon / 5, \quad l=1, \ldots, m .
$$

For each $x \in\left[\alpha, a_{0}\right]$ there is $l, 1 \leqq l \leqq m$ such that $0 \leqq x-x_{r_{l}}<\varepsilon / 5 L$. For any $v, s$ 
we have then

$$
\begin{aligned}
& \int_{-Y}^{Y}\left|V^{(s)}(x, y)-V^{(v)}(x, y)\right| \mathrm{d} y \leqq \int_{-Y}^{Y}\left|V^{(s)}(x, y)-V^{(s)}\left(x_{r_{l}}, y\right)\right| \mathrm{d} y+ \\
& +\int_{-Y}^{Y}\left|V^{(s)}\left(x_{r_{l}}, y\right)-V^{(v)}\left(x_{r_{l}}, y\right)\right| \mathrm{d} y+ \\
& +\int_{-Y}^{Y}\left|V^{(v)}\left(x_{r_{l}}, y\right)-V^{(v)}(x, y)\right| \mathrm{d} y .
\end{aligned}
$$

Using the definition of $V^{(v)}$ we get

$$
\begin{aligned}
& \int_{-Y}^{Y}\left|V^{(v)}(x, y)-V^{(v)}\left(x_{r_{l}}, y\right)\right| \mathrm{d} y \leqq \\
& \leqq \sum_{j \in 2 Z,,\left|j k_{v}\right| \leqq Y} 2 k_{v} \mid\left[\Delta_{1} v^{(i, j)}+\frac{x-x^{(i)}}{h_{v}}\left(\Delta_{1} v^{(i+1, j)}-\Delta_{1} v^{(i, j)}\right)\right]- \\
& -\left[\Delta_{1} v^{\left(i^{\prime}, j\right)}+\frac{x_{\boldsymbol{r}_{1}}-x^{\left(i^{\prime}\right)}}{h_{v}}\left(\Delta_{1} v^{\left(i^{\prime}+1, j\right)}-\Delta_{1} v^{\left(i^{\prime}, j\right)}\right)\right],
\end{aligned}
$$

where $i=\left[x / h_{v}\right], i^{\prime}=\left[x_{r_{l}} / h_{v}\right]$. Hence by force of $(15)$ we have

$$
\begin{aligned}
& \int_{-Y}^{Y}\left|V^{(v)}(x, y)-V^{(v)}\left(x_{r_{l}}, y\right)\right| \mathrm{d} y \leqq \sum_{|j| \leqq Y / k_{v}} 2 k_{v}\left[\left|\Delta_{1} v^{(i, j)}-\Delta_{1} v^{\left(i^{\prime}, j\right)}\right|+\right. \\
& \left.+\left|\Delta_{1} v^{(i+1, j)}-\Delta_{1} v^{(i, j)}\right|+\left|\Delta_{1} v^{\left(i^{\prime}+1, j\right)}-\Delta_{1} v^{\left(i^{\prime}, j\right)}\right|\right] \leqq \\
& \leqq L h_{v}\left(\left[\frac{x}{h_{v}}\right]-\left[\frac{x_{r_{l}}}{h_{v}}\right]\right)+2 L h_{v} \leqq L\left(x-x_{r_{l}}\right)+3 L h_{v}<\frac{2}{5} \varepsilon,
\end{aligned}
$$

for $v$ sufficiently large. Finally, we obtain

$$
\int_{-Y}^{Y}\left|V^{(s)}(x, y)-V^{(v)}(x, y)\right| \mathrm{d} y<\varepsilon,
$$

for $s, v$ sufficiently large. This ends the proof of (16).

Since the convergence in (16) is uniform on $\left[\alpha, a_{0}\right]$ for any $\alpha \in\left(0, a_{0}\right)$ we obtain the almost uniform convergence on $\left[0, a_{0}\right]$. From this we have $\lim _{v, s \rightarrow \infty}\left\|V^{(s)}-V^{(v)}\right\|_{L(Y)}=0$. The completeness of $\left.L^{\prime} Y\right)$ completes the proof of Lemma 9.

Remark 3. If $b_{0}>0$, then it is not necessary to consider the interval $\left[\alpha, a_{0}\right]$, $\alpha>0$, because (16) holds uniformly with respect to $x \in\left[0, a_{0}\right]$.

\section{THE MAIN THEOREM}

Theorem 1. If Assumption $\mathrm{H}$ is satisfied, then there is a function $u \in C\left(\left[-b_{0}, a_{0}\right] \times\right.$ $\times R, R)$ which is a generalized solution of (1), (2).

Proof. It follows from Lemma 7 that there is a sequence $\left\{\left(h_{v}, k_{v}\right)\right\}_{v=1}^{\infty},\left(h_{v}, k_{v}\right) \in \tilde{U}$ such that the sequence $\left\{u^{(v)}\right\}_{v=1}^{\infty}, u^{(v)}=u_{h_{v} k_{v}}$ is uniformly convergent to a function $\tilde{u}$ on $\left[0, a_{0}\right] \times R$. By force of Lemma 9 there is a subsequence of $\left\{V^{(v)}\right\}_{v=1}^{\infty}$ which is convergent in the $L(Y)$ norm to $\bar{v}$. The sequence and its subsequence we denote by the same symbol for simplicity. Let $\tilde{u}(x, y)=\varphi(x, y)$ for $(x, y) \in\left[-b_{0}, 0\right] \times R$. Then the sequence $\left\{\varphi_{h_{v} k_{v}}\right\}_{v=1}^{\infty}$ is uniformly convergent to $\tilde{u}$ on $\left[-b_{0}, 0\right] \times R$. For 
$(x, y) \in\left[0, a_{0}\right] \times R$ we write

$$
\begin{aligned}
& \bar{f}^{(v)}(x, y)=f\left(x, y, u^{(v)}(x, y), u_{(x, y)}^{(v)}, V^{(v)}(x, y)\right), \\
& \bar{w}(x, y)=f\left(x, y, \tilde{u}(x, y), \tilde{u}_{(x, y)}, \bar{v}(x, y)\right) .
\end{aligned}
$$

We will prove that $\lim _{v \rightarrow \infty}\left\|W^{(v)}-\bar{w}\right\|_{L(x)}=0, Y>0$. From

$$
\begin{aligned}
& \left\|W^{(v)}-\bar{w}\right\|_{L(Y)} \leqq\left\|W^{(v)}-\bar{f}^{(v)}\right\|_{L(Y)}+\left\|\bar{f}^{(v)}-\bar{w}\right\|_{L(Y)}, \\
& \lim _{v \rightarrow \infty}\left\|\bar{f}^{(v)}-\bar{w}\right\|_{L(Y)}=0,
\end{aligned}
$$

we see that it is sufficient if we prove

$$
\lim _{v \rightarrow \infty}\left\|W^{(v)}-\bar{f}^{(v)}\right\|_{L(Y)}=0 .
$$

If $\alpha>0$, then for each $x \in\left[\alpha, a_{0}\right]$ there is $i, 0 \leqq i \leqq n_{0}-1$ such that $x \in$ $\in\left[x^{(i)}, x^{(i+1)}\right)$. (If $b_{0}>0$, then we take $x \in\left[0, a_{0}\right]$.) We have then

$$
\begin{aligned}
& \int_{-Y}^{Y}\left|W^{(v)}(x, y)-f^{(v)}(x, y)\right| \mathrm{d} y \leqq \\
& \sum_{j \in 2 Z,\left|j k_{v}\right| \leqq Y} \int_{y(j-1)}^{y^{(j+1)}} \mid \Delta_{0} v^{(i, j-1)}-\bar{f}^{(v)}(x, y)+ \\
& +\frac{y-y^{(j-1)}}{h_{v}}\left[\Delta_{1} v^{(i+1, j)}-\Delta_{1} v^{(i, j)}\right] \mathrm{d} y \leqq \\
& \leqq \\
& \sum_{|j| \leqq Y / k_{v}} \int_{y(j-1)}^{y^{(j+1)}} \mid f\left(x^{(i)}, y^{(j-1)}, \frac{1}{2}\left(v^{(i, j)}+v^{(i, j-2)}\right), u_{(x(i), y(j-1))}^{(v)}, \Delta_{1} v^{(i, j-1)}\right)- \\
& -f^{(v)}(x, y)\left|\mathrm{d} y+\sum_{|j| \leqq Y / k_{v}} \frac{2 k_{v}}{h_{v}}\right| \frac{1}{2}\left(v^{(i, j)}+v^{(i, j-2)}\right)-v^{(i, j-1)} \mid+ \\
& +\sum_{|j| \leqq Y / k_{v}} \frac{\left(2 k_{v}\right)^{2}}{h_{v}}\left|\Delta_{1} v^{(i+1, j)}-\Delta_{1} v^{(i, j)}\right| .
\end{aligned}
$$

Using the Lipschitz condition for $f$, Lemmas $1-3$ and Remark 2 we see that the first component of the right hand side of the above inequality tends to zero if $v \rightarrow \infty$. From Remark 2 we obtain that there is a constant $C_{0}$ such that

and hence

$$
\sum_{|j| \leqq Y / k_{v}} 2 k_{v}\left|\widetilde{\Delta}_{11}^{2} v^{(i, j)}\right| \leqq C_{0},
$$

$$
\sum_{|j| \leqq Y / k_{v}} \frac{2 k_{v}}{h_{v}}\left|\frac{1}{2}\left(v^{(i, j)}+v^{(i, j-2)}\right)-v^{(i, j-1)}\right| \leqq B C_{0} k_{v} .
$$

Futhermore, from Lemma 6 we have

$$
\sum_{|j| \leqq Y / k_{v}} \frac{\left(2 k_{v}\right)^{2}}{h_{v}}\left|\Delta_{1} v^{(i+1, j)}-\Delta_{1} v^{(i, j)}\right| \leqq 2 B L h_{v} .
$$

Finally, we obtain

$$
\lim _{v \rightarrow \infty} \int_{-Y}^{Y}\left|W^{(v)}(x, y)-f^{(v)}(x, y)\right| \mathrm{d} y=0,
$$

almost uniformly with respect to $x \in\left[0, a_{0}\right]$, from which we have (17). 
From Lemmas 1, 2 and from Remark 2 we obtain that $\tilde{u}$ satisfies the Lipschitz condition on $\left[0, a_{0}\right] \times R$, and hence the derivatives $D_{x} \tilde{u}, D_{y} \tilde{u}$ exist a.e. on $\left[0, a_{0}\right] \times$ $\times R$. Since the sequences $\left\{V^{(v)}\right\}_{v=1}^{\infty},\left\{W^{(v)}\right\}_{v=1}^{\infty}$ are equibounded and convegent in the $L(Y)$ norm to $\bar{v}, \bar{w}$ respectively, it follows that $D_{x} \tilde{u}=\bar{w}, D_{y} \tilde{u}=\bar{v}$ a.e. on $\left[0, a_{0}\right] \times$ $\times[-Y, Y]$ for any $Y>0$. Thus $\tilde{u}$ satisfies (1) a.e. on $\left[0, a_{0}\right] \times R$. From the definition of $\tilde{u}$ we see that the initial condition (2) holds. Furthermore, it is easy to prove the existence of a constant $M>0$ such that for any $(x, y) \in\left[0, a_{0}\right] \times R, l \in R$, $l \neq 0$ we have

$$
\begin{array}{ll}
l^{-2}[\tilde{u}(x, y+l)-2 \tilde{u}(x, y)+\tilde{u}(x, y-l)] \leqq M & \text { if } \quad b_{0}=0, \\
l^{-2}[\tilde{u}(x, y+l)-2 \tilde{u}(x, y)+\tilde{u}(x, y-l)] \leqq M / x & \text { if } \quad b_{0}>0 .
\end{array}
$$

Hence $u$ is a generalized solution of (1), (2), which ends the proof.

Remark 4. Using the same methods as in the proof of Theorem 1 we obtain the existence of a generalized solution of the following differential-functional system of first order partial equations

$$
\begin{aligned}
& D_{x} z_{i}(x, y)=f_{i}\left(x, y, z(x, y), z_{(x, y)}, D_{y} z_{i}(x, y)\right), \\
& z_{i}(x, y)=\varphi_{i}(x, y), \quad(x, y) \in\left[-b_{0}, 0\right] \times R,
\end{aligned}
$$

where $i=1, \ldots, m, z=\left(z_{1}, \ldots, z_{m}\right), \varphi=\left(\varphi_{1}, \ldots, \varphi_{m}\right):\left[-b_{0}, 0\right] \times R \rightarrow R^{m}, f=$ $=\left(f_{1}, \ldots, f_{m}\right):\left[0, a_{0}\right] \times R \times R^{m} \times C\left(B, R^{m}\right) \times R \rightarrow R^{m}$.

\section{References}

[1] P. Bassanini: Su una recente dimostrazione cirza il problema di Cauchy per sistemi quasi lineari iperbolici, Boll. Un. Mat. Ital. (5) 13-B (1976), 322-335.

[2] P. Bassanini: On a recent proof concerning a boundary value problem for quasilinear hyperbolic systems in the Schauder canonic form, Boll. Un. Mat. Ital. (5) 14-A (1977), 325-332.

[3] P. Bassanini: Iterative methods for quasilinear hyperbolic systems, Boll. Un. Mat. Ital. (6) 1-B (1982), 225-250.

[4] P. Bassanini, M. C. Salvatori: Un problema ai limiti per sistemi integrodifferenziali non lineari di tipo iperbolico, Boll. Un. Mat. Ital. (5) 18-B (1981), 785-798.

[5] P. Brandi, R. Ceppitelli: On the existence of the solution of a nonlinear functional partial differential equations of the first order, Atti. Sem. Mat. Fis. Univ. Modena 29 (1980), $166-186$.

[6] P. Brandi, R. Ceppitelli: Existence, uniqueness and continuous dependence for a first order non linear partial differential equation in a hereditary structure, Ann. Polon. Math. 47 (1986), 121-136.

[7] L. Cesari: A boundary value problem for quasilinear hyperbolic systems, Riv. Mat. Univ. Parma 3 (1974), 107-131.

[8] L. Cesari: A boundary value problem for quasilinear hyperbolic systems in the Schauder canonic form, Ann. Sc. Norm. Sup. Pisa (4) 1 (1974), 311-358.

[9] M. Cinquini-Cibrario, S. Cinquini: Equazioni alle derivate parziali di tipo iperbolico, Cremonese, Roma 1964. 
[10] Z. Kamont: On the Cauchy problem for nonlinear partial differential-functional equations of the first order, Math. Nachr. 88 (1979), 13-29.

[11] Z. Kamont: On the estimation of the existence domain for solutions of a nonlinear partial differential-functional equation of the first order, Glasnik Mat. 13 (1978), 277-291.

[12] Z. Kamont: Existence of solutions of first order partial differential-functional equations, Ann. Soc. Math. Polon., Ser. I: Comm. Math. 25 (1985), 249-263.

[13] Z. Kamont, J. Turo: On the Cauchy problem for quasilinear hyperbolic system of partial differential equations with a retarded argument, Boll. Un. Mat. Ital. (6) 4-B (1985), 901-916.

[14] Z. Kamont, J. Turo: A boundary value problem for quasilinear hyperbolic systems with a retarded argument, Ann. Polon. Math. 47 (1987), 347-360.

[15] Z. Kamont, S. Zacharek: On the existence of weak solutions of nonlinear first order partial differential equations in two independent variables, Boll. Un. Mat. Ital. (6) 5-B (1986), $851-879$.

[16] S. N. Kruzhkov: Generalized solutions of non linear first order partial differential equations (Russian), Mat. Sb. 70 (1966), 394-415.

[17] $O$. A. Oleynik: Discontinuous solutions of non linear differential equations (Russian), Usp. Mat. Nauk. 12, 3 (1957), 3-73.

[18] P. Pucci: Problemi ai limiti per sistemi di equazioni iperboliche, Boll. Un. Mat. Ital. (5) 16-B (1979), 87-99.

[19] B. L. Rozhdestvenskij, N.N.Yanenko: Systems of quasilinear equations and their applications to gas dynamics, Providence, Rhode Island 1983.

[20] J. Turo: A boundary value problem for quasilinear hyperbolic systems of hereditary partial differential equations, Atti. Sem. Mat. Fis. Univ. Modena 34 (1985-86), 15-34.

[21] J. Turo: On some class of quasilinear hyperbolic systems of partial differential-functional equations of the first order, Czech. Math. J. 36 (111) (1986), 185-197.

[22] J. Turo: Generalized solutions to functional partial differential equations of the first order, Zesz. Nauk. Polit. Gd. 427, Mat. 14 (1988), 3-98.

Author's address: Institute of Mathematics, University of Gdańsk, ul. Wita Stwosza 57, 80-952 Gdańsk, Poland. 